\title{
Infinitely many precomplete with respect to parametric expressibility classes of formulas in a provability logic of propositions
}

\author{
Andrei Rusu
}

\begin{abstract}
In the present paper we consider a non-tabular extension $L$ of the well-known propositional provability logic $G L$ together with the notion of parametric expressibility of formulas in a logic proposed by A. V. Kuznetsov. We prove that there are infinitely many precomplete with respect to parametric expressibility classes of formulas in the above mentioned logic $L$.
\end{abstract}

\section{Introduction}

We consider an extension $L$ of the propositional provability logic of GödelLöb [1]. In 1921 E. Post analysed the possibbility to get a formula from other formulas by means of superpositions $[2,3]$ and proved that there is a numerable collection of closed with respect to superpositions classes of boolean functions, among which only 5 of them are maximal with respect to inclusion. A. V. Kuznetsov have generalized the notion of superposition of functions to the case of formulas and put into consideration the notion of parametric expressibility of a formula via a system of formulas in a given logic $[4,5,6]$ and proved there are finitely many precomplete with respect to parametric

Key Words: parametric expressibility of formulas, modal logic, diagonalizable algebra, precomplete classes of formulas with respect to parametric expressibility, extensions of logics, 08A99.

2010 Mathematics Subject Classification: Primary 03F45, 06E25, 03G25; Secondary

Received: November, 2013

Revised: January, 2014

Accepted: February, 2014 
expressibility classes of formulas in the general 2-valued and 3-valued logics. Later on it was proved in [7] there are finitely many closed with respect to parametric expressibility classes of formulas in the general $k$-valued logic (these classes are reffered in [7] to as primitive positive clones). In the present paper we consider formulas of an extension $L$ of the propositional provability logic $G L$ and prove there are infinitely many precomplete with respect to parametric expressibility classes of formulas in $L$. The proof uses the fact that the above mentioned logic $L$ coincides with a logic of a Magari's algebra defined futher.

\section{Definitions and notations}

Propositional provability logic $G L[1]$. The calculus $G$ of the propositional provability logic $G L$ is based on formulas built as usual from propositional variables $p, q, r, p_{1}, q_{i}, r_{j} \ldots$, logical connectives $\&, \vee, \supset, \neg, \Delta$ and auxiliary symbols of left and right parantheses ( and ). Let Form denote the set of all formulas of the calculus $G$. The variables occuring in the formula $F$ we denote by $\operatorname{Var}(F)$. Axioms of $G L$ are the axioms of the classical logic of propositions together with the following three formulas:

$$
\begin{aligned}
\Delta(p \supset q) & \supset(\Delta p \supset \Delta q), \\
\Delta p & \supset \Delta \Delta p, \\
\Delta(\Delta p \supset p) & \supset \Delta p .
\end{aligned}
$$

The rules of inference are the well-known rules of modus ponens and substitution supplimented with the rule of necessitation:

$$
\frac{A}{\Delta A}
$$

The notion of theorem (deductible formula) in the calculus $G$ is defined as usual based on axioms and inference rules of $G$. Denote all theorems of $G$ by $T h$. Then, the propositional provability logic $G L$ of $G$ is considered the pair (Form, Th). Since set Form is known traditionally by logic $G L$ is understoond the set $T h$. Any set of formulas $L$ containing $G L$ and closed with respect to rules of inference of $G$ is said to be an extension of $G L$.

Denote by $p \sim q$ and $\Delta^{2} p, \ldots, \Delta^{n+1} p, \ldots(n=1,2, \ldots)$ the corresponding formulas $(\neg p \vee q) \&(\neg q \vee p)$ and $\Delta(\Delta p), \ldots, \Delta\left(\Delta^{n} p\right), \ldots$. Denote by $\square p$ the formula $p \& \Delta p$ and let $\nabla p$ means formula $\square \neg \square \neg \square p$.

Parametric expressibility of formulas [4]. Suppose in the logic $L$ we can define the equivalence of two formulas. The formula $F$ is said to be (explicitly) expressible via a system of formulas $\Sigma$ in the logic $L$ if $F$ can be obtained from any variables and formulas of $\Sigma$ using two rules: 
1. the rule of weak substitution, which allows to to pass from two formulas, let say $A$ and $B$, to the result of substitution of one of them, $B$, in another one, $A$, in place of every occurence of any fixed variable $p$ (the result of substitution we denote as $A[p / B]$, or $A[B]$ )

$$
\frac{A, B}{A[p / B]}
$$

2. if we already get formula $A$ and we know $A$ is equivalent in $L$ to formula $B$, then we can consider we also have formula $B$

$$
\frac{A, A \sim B}{B} .
$$

The formula $F$ is said to bbe parametrically expressible via the system of formulas $\Sigma$ in the logic $L$ if there exist variables $q_{1}, \ldots, q_{s}, q$ not occuring in $F$, formulas $D_{1}, \ldots, D_{s}$, formulas $B_{1}, \ldots, B_{m}$ and $C_{1}, \ldots, C_{m}$ such that $B_{1}, \ldots, B_{m}$ and $C_{1}, \ldots, C_{m}$ are explicitly expressible in $L$ via $\Sigma$ and the following first-order formulas are valid:

$$
\begin{aligned}
(F=q) \Rightarrow & \left({ }_{i=1}^{\wedge_{i=1}^{m}}\left(B_{i}=C_{i}\right)\right)\left[q_{1} / D_{1}\right] \ldots\left[q_{s} / D_{s}\right], \\
& \left(\bigwedge_{i=1}^{m}\left(B_{i}=C_{i}\right)\right) \Rightarrow(F=q) .
\end{aligned}
$$

The system of formulas $\Sigma$ is said to be complete with respect to parametric expressibility in the logic $L$ if any formula of the calculus of $L$ is parametrically expressible via formulas of $\Sigma$. The system $\Sigma$ is called precomplete with respect to parametrical expressibility in the logic $L$ if it is not complete in $L$, but for any formula $F$, which is not parametrically expressible via formulas $\Sigma$, the system $\Sigma \cup\{F\}$ is already parametrically complete in $L$.

Magari's algebras. A Magari's algebra [8] $\mathfrak{D}$ is a boolean algebra $\mathfrak{A}=$ $(A ; \&, \vee, \supset, \neg, \mathbb{O}, \mathbb{1})$ with an additional operator $\Delta$ satisfying the following relations:

$$
\begin{aligned}
\Delta(x \supset y) & \leq \Delta x \supset \Delta y, \\
\Delta x & \leq \Delta \Delta x, \\
\Delta(\Delta x \supset x) & =\Delta x, \\
\Delta \mathbb{1} & =\mathbb{1},
\end{aligned}
$$

where $\mathbb{1}$ is the unit of $\mathfrak{A}$.

We consider the Magari's algebra $\mathfrak{M}=(M ; \&, \vee, \supset, \neg, \Delta)$ of all infinite binary sequences of the type $\alpha=\left(\mu_{1}, \mu_{2}, \ldots\right), \mu_{i} \in\{0,1\}, i=1,2, \ldots$ The 
boolean operations \&, $\vee, \supset, \neg$ over elements of $M$ are defined componentwise, and the operation $\Delta$ over element $\alpha$ we define by the equality $\Delta \alpha=$ $\left(1, \nu_{1}, \nu_{2}, \ldots\right)$, where $\nu_{i}=\mu_{1} \& \cdots \& \mu_{i}$. Consider the subalgebra $\mathfrak{M}^{*}$ of $\mathfrak{M}$ generated by its zero $\mathbb{O}$ element $(0,0, \ldots)$. Remark the unite $\mathbb{1}$ of the algebra $\mathfrak{M}^{*}$ is the element $(1,1, \ldots)$.

Interpreting logical connectives of a formula $F$ by corresponding operations on a Magari's algebra $\mathfrak{D}$ we can evaluate $F$ on any algebra $\mathfrak{D}$. If for any evaluation of variable of $F$ by elements of $\mathfrak{D}$ the resulting value of the formula $F$ on $\mathfrak{D}$ is $\mathbb{1}$ they say $F$ is valid on $\mathfrak{D}$. The set of all valid formulas on a given Magari's algebra $\mathfrak{D}$ is known to form an extension of the logic $G L$ [9].

They say formula $F\left(p_{1}, \ldots, p_{n}\right)$ conserves on the Magari's algebra $\mathfrak{D}$ the relation $R\left(x_{1}, \ldots, x_{m}\right)$ if for any elements $\alpha_{11}, \ldots, \alpha_{m n}$ of $\mathfrak{D}$ the relations

$$
R\left(\alpha_{11}, \ldots, \alpha_{m 1}\right), \ldots, R\left(\alpha_{1 n}, \ldots, \alpha_{m n}\right)
$$

implies

$$
R\left(F\left(\alpha_{11}, \ldots, \alpha_{1 n}\right), \ldots, F\left(\alpha_{m 1}, \ldots, \alpha_{m n}\right)\right)
$$

Let $\alpha \in \mathfrak{D}$. Obviously, formula $F\left(p_{1}, \ldots, p_{n}\right)$ conserves the relation $x=\alpha$ on $\mathfrak{D}$ if $F(\alpha, \ldots, \alpha)=\alpha$. According to A. F. Danil'čenco [6], the set of all formulas conserving the relation $x=\alpha$ on an arbitrary $k$-element set is closed with respect to parametrical expressibility.

\section{Preliminary results}

We start by validating some useful properties of the formulas $\square p, \Delta p$ and $\nabla p$.

Proposition 1. Let $x, y$ arbitrary elements of $\mathfrak{M}^{*}$. Then:

$$
\begin{gathered}
\square x \geq \Delta \mathbb{O}, \text { if and only if } \nabla x=\mathbb{1} \\
\square x=\mathbb{0}, \text { if and only if } \nabla x=\mathbb{0} \\
\text { For any } x, y, \text { either } \square x \leq \square y \text {, or } \square y \leq \square x \\
\quad \Delta x=\Delta \square x \\
\quad \nabla \mathbb{O}=\mathbb{O}, \nabla \mathbb{1}=\mathbb{1} \\
\square x \geq \Delta \mathbb{0} \text { if and only if } \square \neg x=\mathbb{0} \\
\square x=\mathbb{0} \text { if and only if } \square \neg x \geq \Delta \mathbb{0}
\end{gathered}
$$

Proof. The proof is almost obvious by construction of the algebra $\mathfrak{M}^{*}$.

Let us mention the following observation:

Remark 1. Any formula $F$ is parametrically expressible in the logic $L \mathfrak{D}$ of any Magari's algebra $\mathfrak{D}$ via formulas $p \& q, p \vee q, p \supset q, \neg p, \Delta q$. 
Let us consider on $\mathfrak{D}$ the following formulas (16) and (17), denoted by $F_{\neg}(p, q, r)$ and $F_{\Delta}(p, q, r)$ correspondingly:

$$
\begin{gathered}
(\nabla \neg(p \sim q) \&((\neg p \sim q) \sim r)) \vee\left(\nabla(p \sim q) \& \neg \Delta^{i} \mathbb{O}\right) \\
(\nabla q \&((\Delta p \sim q) \sim r)) \vee\left(\neg \nabla q \& \neg \Delta^{i} \mathbb{O}\right)
\end{gathered}
$$

Proposition 2. Let arbitrary $\alpha, \beta, \xi \in \mathfrak{M}^{*}$. If $\neg \alpha=\beta$ on $\mathfrak{M}^{*}$, then

$$
F_{\neg}[p / \alpha, q / \beta, r / \xi]=\xi
$$

on $\mathfrak{M}^{*}$.

Proof. Since $\neg \alpha=\beta$ we get $\alpha \sim \beta=\mathbb{O}, \neg(\alpha \sim \beta)=\mathbb{1}$ and by (13) we have

$$
\nabla(\alpha \sim \beta)=\mathbb{0}, \nabla \neg(\alpha \sim \beta)=\mathbb{1},
$$

which implies

$$
F_{\neg}[p / \alpha, q / \beta, r / \xi]=(\mathbb{1} \&(\mathbb{1} \sim \xi)) \vee\left(\mathbb{O} \& \neg \Delta^{i} \mathbb{O}\right)=\xi
$$

Proposition 3. Let arbitrary $\alpha, \beta, \xi \in \mathfrak{M}^{*}$. If $\neg \alpha \neq \beta$ on $\mathfrak{M}^{*}$ and $\xi \neq \neg \Delta^{i} \mathbb{O}$, then

$$
F_{\neg}[p / \alpha, q / \beta, r / \xi] \neq \xi
$$

on $\mathfrak{M}^{*}$.

Proof. Since $\neg \alpha \neq \beta$ we get $\neg \alpha \sim \beta \neq \mathbb{1}, \alpha \sim \beta \neq \mathbb{0}$ We distinguish two cases: 1) $\square(\alpha \sim \beta)=\mathbb{0}$, and 2) $\square(\alpha \sim \beta) \geq \Delta \mathbb{0}$.

In case 1) by (15), (9) and (10) we get $\square \neg(\alpha \sim \beta) \geq \Delta \mathbb{0}, \nabla \neg(\alpha \sim \beta)=$ $\mathbb{1}$, and $\nabla(\alpha \sim \beta)=\mathbb{0}$, which implies

$$
\begin{aligned}
F_{\neg}[p / \alpha, q / \beta, r / \xi] & =(\nabla \neg(\alpha \sim \beta) \&((\neg \alpha \sim \beta) \sim \xi)) \vee\left(\nabla(\alpha \sim \beta) \& \neg \Delta^{i} \mathbb{O}\right. \\
& =\left(\mathbb{1} \&((\neg \alpha \sim \beta) \sim \xi) \vee\left(\mathbb{0} \& \neg \Delta^{i} \mathbb{O}\right)=(\neg \alpha \sim \beta) \sim \xi \neq \xi,\right.
\end{aligned}
$$

Thus the first case is already examined.

Now consider the second case, when $\square x \geq \Delta \mathbb{0}$. Again, since $\neg \alpha \neq \beta$ by (9), (10) and (14) we obtain $\square \neg(\alpha \sim \beta)=\mathbb{O}, \nabla \neg(\alpha \sim \beta)=\mathbb{0}, \nabla(\alpha \sim \beta)=\mathbb{1}$.

Then,

$$
\begin{aligned}
F_{\neg}[p / \alpha, q / \beta, r / \xi] & =(\nabla \neg(\alpha \sim \beta) \&((\neg \alpha \sim \beta) \& \xi)) \vee\left(\nabla(\alpha \sim \beta) \& \neg \Delta^{i} \mathbb{O}\right) \\
& =(\mathbb{O} \&((\neg \alpha \sim \beta) \& \xi)) \vee\left(\mathbb{1} \& \neg \Delta^{i} \mathbb{O}\right)=\neg \Delta^{i} \mathbb{O} \neq \xi .
\end{aligned}
$$


Proposition 4. Let arbitrary $\alpha, \beta, \eta \in \mathfrak{M}^{*}$ such that $\Delta \alpha=\beta$. Then

$$
F_{\Delta}[p / \alpha, q / \beta, r / \eta]=\eta
$$

Proof. Since $\Delta \alpha \geq \mathbb{O}$ and $\Delta \alpha=\beta$ we have $\square \beta \geq \Delta \mathbb{O}, \Delta \alpha \sim \beta=\mathbb{1}$ and by (9) we get $\nabla \beta=\mathbb{1}, \neg \nabla \beta=\mathbb{0}$. These ones imply the following relations:

$$
\begin{aligned}
F_{\Delta}[p / \alpha, q / \beta, r / \eta] & =(\nabla \beta \&((\Delta \alpha \sim \beta) \sim \eta)) \vee\left(\neg \nabla \beta \& \neg \Delta^{i} \mathbb{0}\right) \\
& =(\mathbb{1} \&(\mathbb{1} \sim \eta)) \vee\left(\mathbb{O} \& \neg \Delta^{i} \mathbb{O}\right)=\mathbb{1} \sim \eta=\eta
\end{aligned}
$$

Proposition 5. Let arbitrary $\alpha, \beta, \eta \in \mathfrak{M}^{*}$ such that $\Delta \alpha \neq \beta$ and $\eta \neq \neg \Delta^{i} \mathbb{O}$. Then

$$
F_{\Delta}[p / \alpha, q / \beta, r / \eta) \neq \eta
$$

Proof. We consider 2 cases: 1) $\square \beta=\mathbb{D}$, and 2) $\square \beta \geq \Delta \mathbb{0}$.

Suppose $\square \beta=\mathbb{O}$. In view of $(10)$ we have $\nabla \beta=\mathbb{O}$ and $\neg \nabla \beta=\mathbb{1}$. Subsequently,

$$
\begin{aligned}
F_{\Delta}[p / \alpha, q / \beta, r / \eta] & =(\nabla \beta \&((\Delta \alpha \sim \beta) \sim \eta)) \vee\left(\neg \nabla \beta \& \neg \Delta^{i} \mathbb{O}\right) \\
& =(\mathbb{O} \&((\Delta \alpha \sim \beta) \sim \eta)) \vee\left(\mathbb{1} \& \neg \Delta^{i} \mathbb{O}\right) \\
& =0 \vee \neg \Delta^{i} \mathbb{O}=\neg \Delta^{i} \mathbb{O} \neq \eta
\end{aligned}
$$

Suppose now $\square \beta \geq \Delta \mathbb{0}$. Let us note $\Delta \alpha \sim \beta \neq \mathbb{1}$. Then considering (9) we get

$$
\begin{aligned}
F_{\Delta}[p / \alpha, q / \beta, r / \eta] & =(\nabla \beta \&((\Delta \alpha \sim \beta) \sim \eta)) \vee\left(\neg \nabla \beta \& \neg \Delta^{i} \mathbb{O}\right) \\
& =(\mathbb{1} \&((\Delta \alpha \sim \beta) \sim \eta)) \vee\left(\mathbb{O} \& \neg \Delta^{i} \mathbb{O}\right) \\
& =(\Delta \alpha \sim \beta) \sim \eta \neq \eta
\end{aligned}
$$

Proposition 6. Let arbitrary $\alpha, \xi \in \mathfrak{M}^{*}$. Then

$$
F_{\neg}[p / \alpha, q / \alpha, r / \xi]=\neg \Delta^{i} \mathbb{O} .
$$

Proof. Let us calculate $F_{\neg}[p / \alpha, q / \alpha, r / r]$. By (13) we obtain imidiately:

$$
\begin{aligned}
F_{\neg}[p / \alpha, q / \alpha, r / \xi] & =(\nabla \neg(\alpha \sim \alpha) \&((\neg \alpha \sim \alpha) \& \xi)) \vee\left(\nabla(\alpha \sim \alpha) \& \neg \Delta^{i} \mathbb{O}\right) \\
& =(\mathbb{O} \&(\mathbb{O} \& \xi)) \vee\left(\mathbb{1} \& \neg \Delta^{i} \mathbb{O}\right)=\neg \Delta^{i} \mathbb{O} .
\end{aligned}
$$


Proposition 7. Let arbitrary $\alpha, \eta \in \mathfrak{M}^{*}$ and $\square \alpha=\mathbb{O}$. Then

$$
F_{\Delta}[p / \alpha, q / \alpha, r / \eta]=\neg \Delta^{i} \mathbb{O} .
$$

Proof. Taking into account (10) we have

$$
\begin{aligned}
F_{\Delta}[p / \alpha, q / \alpha, r / \eta] & =(\nabla \alpha \&((\Delta \alpha \sim \alpha) \sim \eta)) \vee\left(\neg \nabla \alpha \& \neg \Delta^{i} \mathbb{O}\right) \\
& =(\mathbb{O} \&((\Delta \alpha \sim \alpha) \sim \eta)) \vee\left(\mathbb{1} \& \neg \Delta^{i} \mathbb{O}\right)=\mathbb{O} \vee \neg \Delta^{i} \mathbb{O}=\neg \Delta^{i} \mathbb{O} .
\end{aligned}
$$

\section{Some properties of some classes of formulas in $L \mathfrak{M}^{*}$}

Consider the class $K_{i}, i=1,2, \ldots$, of all formulas of $G L$ which conserves the relation $x=\neg \Delta^{i} \mathbb{O}$ on $\mathfrak{M}^{*}$. For example, the class $K_{1}$ is defined by the relation $x=(0,1,1,1, \ldots)$.

Remark 2. The formulas $\square p, p \& q, p \vee q, \neg \Delta^{i} \mathbb{O} \in K_{i}$, and $\neg p, \Delta p \notin K_{i}$.

As a consequence from [6] we have the following remark.

Remark 3. The class $K_{i}, i=1,2, \ldots$, of formulas is closed with respect to parametric expressibility of formulas in the logic L $L \mathfrak{M}^{*}$.

Remark 4. Since $K_{i}$ is closed relativ to parametric expressibility the formulas $\neg p$ and $\Delta p$ can not be expressed parametrically via formulas of $K_{i}, K_{i}$ in $L \mathfrak{M}^{*}$, so $K_{i}$ is not complete relative to parametric expressibility of formulas in $L \mathfrak{M}^{*}$.

Remark 5. By propositions 6 and 7 we have the earlier defined formulas $F_{\neg}(p, q, r)$ and $F_{\Delta}(p, q, r)$ are in $K_{i}$.

Lemma 1. Consider an arbitrary formula $F\left(p_{1}, \ldots, p_{k}\right) \notin K_{i}$. Then formulas $\Delta p \quad$ and $\neg p$ are parametrically expressible via formulas from $K_{i} \cup\left\{F\left(p_{1}, \ldots, p_{k}\right)\right\}$

Proof. Let us note, since $F \notin K_{i}$, we have $F\left(\neg \Delta^{i} \mathbb{0}, \ldots, \neg \Delta^{i} \mathbb{O}\right) \neq \neg \Delta^{i} \mathbb{0}$. Consider formulas $F_{\neg}^{\prime}$ and $F_{\Delta}^{\prime}$ defined by formulas (18) and (19):

$$
\begin{gathered}
(\nabla \neg(p \sim q) \&((\neg p \sim q) \\
\left.\left(\nabla F\left(\neg \Delta^{i} \mathbb{O}, \ldots, \neg \Delta^{i} \mathbb{O}\right)\right)\right) \vee\left(\nabla(p \sim q) \& \neg \Delta^{i} \mathbb{O}\right) \\
\left.\left((\Delta p \sim q) \sim F\left(\neg \Delta^{i} \mathbb{O}, \ldots, \neg \Delta^{i} \mathbb{O}\right)\right)\right) \vee\left(\neg \nabla q \& \neg \Delta^{i} \mathbb{O}\right)
\end{gathered}
$$

and examine first-order formulas containing only formulas from $K_{i} \cup\{F\}$ :

$$
\left(F_{\neg}^{\prime}(p, q)=F\left(\neg \Delta^{i} \mathbb{O}, \ldots, \neg \Delta^{i} \mathbb{O}\right)\right) \text { and }\left(F_{\Delta}^{\prime}(p, q)=F\left(\neg \Delta^{i} \mathbb{O}, \ldots, \neg \Delta^{i} \mathbb{O}\right)\right)
$$

Let us note by propositions 2 and 3 we have $(\neg p=q)$ if and only if $\left(F_{\neg}^{\prime}(p, q)=\right.$ $\left.F\left(\neg \Delta^{i} \mathbb{O}, \ldots, \neg \Delta^{i} \mathbb{O}\right)\right)$ and according to propositions 4 and 5 we get $(\Delta p=q)$ if and only if $\left.F_{\Delta}^{\prime}(p, q)=F\left(\neg \Delta^{i} \mathbb{O}, \ldots, \neg \Delta^{i} \mathbb{O}\right)\right)$.

Lemma is proved. 


\section{Main result}

Theorem 1. Consider the extension $\mathrm{LM}^{*}$ of the propositional provability logic $G L$. Then there are infinitely many precomplete with respect to parametrical expressibility classes of formulas in $L_{\mathfrak{M}^{*}}$.

Proof. Consider classes of formulas $K_{1}, K_{2}, \ldots$ each of which preserve on the algebra $\mathfrak{M}^{*}$ the corresponding predicates $x=\neg \Delta \mathbb{O}, x=\neg \Delta^{2} \mathbb{O}, \ldots$ According to remark 2 these classes are two by two distinct, and by lemma 1 these classes are precomplete relative to parametric expressibility of formulas in $L \mathfrak{M}^{*}$.

Acknowledgement: The publication of this article was supported by the grant PN-II-ID-WE-2012-4-169 of the Workshop "A new approach in theoretical and applied methods in algebra and analysis".

\section{References}

[1] Solovay R.M., Provability interpretations of modal logic // Israel J. Math., 1975, 25, p. 287 - 304.

[2] Post E.L. Introduction to a general theory of elementary propositions // Amer. J. Math., 1921, v. 43, p. 163 - 185.

[3] Post E.L. Two-valued iterative systems of mathematical logic. Princeton, 1941.

[4] Kuznetsov A. V., On detecting non-deducibility and non-expressibility in: Locical deduction, Nauka, Moscow (1979), 5-33 (in russian)

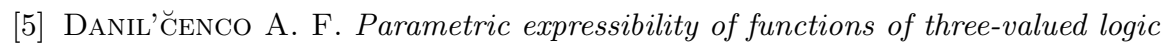
// Algebra i Logika, 16 (1977), pp.397-416 (in russian)

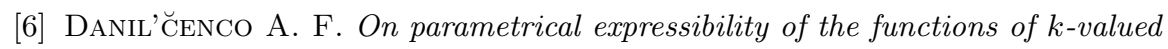
logic // Colloq. Math. Soc. Janos Bolyai, 28, North-Holland, 1981, pp. 147-159.

[7] Burris S., Willard R. Finitely many primitive positive clones // Proceedings of the American Mathematical Society, 101, no. 3, 1987, pp. 427-430.

[8] Magari R., The diagonalizable algebras (the algebraization of the theories which express Theor.: II) // Boll. Unione Mat. Ital. , 12 (1975) (suppl. fasc 3) pp. 117-125.

[9] Maksimova, L.L. Continuum of normal extensions of the modal logic of provability with the interpolation property // Sib. Math. J. 30, No.6, 935-944 (1989) 
Andrei RUSU,

Department of Mathematics and Computer Science,

Ovidius University of Constanta,

Bdul Mamaia 124, 900527 Constanţa, România.

Email: agrusu@univ-ovidius.ro

Information Society Development Institute,

Academy of Sciences of Moldova,

Str. Academiei 5A, MD2028 Chişinău, Republica Moldova.

Email: andrei.rusu@idsi.md 